\title{
PHILIPPE SÉCHER
}

\section{Étude spectrale du système différentiel $2 \times 2$ associé à un problème d'élasticité linéaire}

\author{
Annales de la faculté des sciences de Toulouse $6^{e}$ série, tome $7, \mathrm{n}^{\circ} 4$ \\ (1998), p. 699-726 \\ <http://www.numdam.org/item?id=AFST_1998_6_7_4_699_0>
}

(C) Université Paul Sabatier, 1998, tous droits réservés.

L'accès aux archives de la revue «Annales de la faculté des sciences de Toulouse » (http://picard.ups-tlse.fr/ annales/) implique l'accord avec les conditions générales d'utilisation (http://www.numdam.org/conditions). Toute utilisation commerciale ou impression systématique est constitutive d'une infraction pénale. Toute copie ou impression de ce fichier doit contenir la présente mention de copyright.

\section{NumDam}

Article numérisé dans le cadre du programme Numérisation de documents anciens mathématiques http://www.numdam.org/ 


\title{
Étude spectrale du système différentiel $2 \times 2$ associé à un problème d'élasticité linéaire ${ }^{(*)}$
}

\author{
PHILIPPE SÉCHER ${ }^{(1)}$
}

\begin{abstract}
RÉSUMÉ. - On construit les fonctions propres généralisées d'un système différentiel $2 \times 2$ sur $\mathbb{R}+$. Cet opérateur est lié à l'élasticité linéaire sur un espace $\mathbb{R}_{+}^{n}, n \geq 2$, homogène isotrope avec contrainte nulle sur le bord. Pour cette construction, on étudie la condition de Lopatinski uniforme. On utilise ces fonctions pour construire la transformation unitaire de Fourier associée à notre opérateur autoadjoint.
\end{abstract}

Abstract. - We constructed the generalized eigenfunction of differential system $2 \times 2$ in $\mathbb{R}^{+}$. This operator is a reduction of the problem governing the propagation of elastic waves in homogeneous and isotropic space $\mathbb{R}_{+}^{n}, n \geq 2$, with free boundary condition. For this construction we study the uniform Lopatinski condition. We use this fonctions to construct an unitary Fourier transform associated to this self adjoint operator.

\section{Introduction}

Considérons l'opérateur

$$
A=\left(\begin{array}{cc}
c^{2} D^{2}+1 & \left(c^{2}-1\right) D \\
\left(c^{2}-1\right) D & c^{2}+D^{2}
\end{array}\right) \quad \text { avec } c>1
$$

muni du domaine

$$
\mathcal{D}=\left\{u=\left(\begin{array}{l}
u_{1} \\
u_{2}
\end{array}\right) \in H^{2}\left(\mathbb{R}^{+}\right)|a(u)|_{x=0}=\left.b(u)\right|_{x=0}=0\right\}
$$

(*) Reçu le 13 mai 1997, accepté le 20 janvier 1998

(1) Département de Mathématiques, Université de Nantes, 2 rue de la Houssinière, B. P. 92208, F-44322 Nantes Cedex 03 (France)

E-mail : secher@math.univ-nantes.fr 
où l'on note

$$
\begin{aligned}
a(u) & =u_{1}+D u_{2} \\
b(u) & =c^{2} D u_{1}+\left(c^{2}-2\right) u_{2} \\
D & =\frac{1}{i} \frac{\partial}{\partial x} .
\end{aligned}
$$

Dans cet article on commence par montrer l'origine physique de l'opérateur $A, \mathcal{D}$. Elle est liée à l'étude spectrale de l'opérateur d'élasticité linéaire dans un demi-espace $\mathbb{R}_{+}^{n}$ homogène et isotrope. Pour cette étude on utilise des techniques de scattering. Des études du noyau de scattering sur le complémentaire d'un borné dans le cas non isotrope ont été effectuées par (SSo) et [So]. Ici on recherche la résolution spectrale. Dans le cas de $\mathbb{R}_{+}^{3}$ une étude a été effectuée par [DG]. En utilisant des techniques similaires à celles employées par Wilcox [W] pour l'étude du Laplacien dans un espace stratifié, on montre que l'étude sur $\mathbb{R}_{+}^{n}$ découle de celle de $A, \mathcal{D}$. La principale difficulté rencontrée dans l'application des techniques de scattering, provient de la condition de Neumann sur le bord. Elle fait apparaître du spectre ponctuel pour $A, \mathcal{D}$ contrairement à ce qui se passe dans le cas du Laplacien.

Pour résoudre ce problème au bord, on cherche les fonctions propres généralisées bornées, cela suffit pour calculer la résolution spectrale, voir [Sh]. Pour les construire, on étudie la condition de Lopatinski uniforme comme définie par Chazarain et Piriou [CP]. Cette construction, qui me semble nouvelle, constitue le principal intérêt de ce travail.

Pour finir, on calcule la résolution spectrale de $A, \mathcal{D}$ à partir de transformations associées à chaque fonction propre généralisées.

\section{Origine physique de l'étude}

L'étude de $A, \mathcal{D}$ correspond à un problème d'élasticité linéaire sur un demi-plan isotrope et homogène avec contrainte nulle sur le bord (cf. [LP]). Plus généralement, cet opérateur intervient dans tout problème d'élasticité linéaire sur un demi-espace $\mathbb{R}_{+}^{n}(n \geq 3)$ homogène isotrope, avec condition de Neumann sur le bord.

En effet, notons $\rho$ la densité volumique et $\lambda, \mu$ les paramètres de Lamé qui sont alors constants. Ils vérifient les conditions suivantes :

$$
\begin{gathered}
\rho>0, \quad \mu>0 \quad \text { et enfin } n \lambda+2 \mu>0 . \\
-700-
\end{gathered}
$$


Les deux dernières relations étant liées à la positivité de l'énergie associée à l'opérateur d'élasticité linéaire défini par

$$
\Delta_{e}=-\left(\frac{\mu}{\rho} \Delta+\frac{\lambda+\mu}{\rho} \operatorname{grad} \operatorname{div}\right),
$$

on fait opérer $\Delta_{e}$ sur des fonctions $u: \mathbb{R}_{+}^{n} \rightarrow \mathbb{R}^{n}$. On recherche $u$ dans $\mathbf{H}=L^{2}\left(\mathbb{R}_{+}^{n}\right)$.

La condition de Neumann sur le bord $\left\{x_{1}=0\right\}$ s'écrit

$$
(N(u))_{i}=\left.\sum_{j=1}^{n} \sigma_{i, j}(u)\right|_{x_{1}=0}=0
$$

où $\sigma_{i, j}(u)=\lambda \operatorname{div} u \delta_{i, j}+\mu\left(\partial_{x_{i}} u_{j}+\partial_{x_{j}} u_{i}\right)$ désigne le tenseur de contrainte.

On étudie le problème dans l'espace $\mathbb{R}_{+}^{n}$, homogène isotrope soumis à une force extérieure exercée par une source de densité $F$. Le déplacement $u(x, t)$, au temps $t$ et au point $x$ dû à la source $F$ vérifie alors l'équation

$$
\forall x \in \mathbb{R}_{+}^{n}, \forall t \in \mathbb{R}, \quad\left(\Delta_{e}+\frac{\partial^{2}}{\partial t^{2}}\right) u(x, t)=F(x, t) .
$$

Dans la suite $u(x, t)$ désigne un élément de $\mathbb{C}^{n}$ dont la partie réelle est le déplacement. Si on cherche $u(x, t)$ sous forme de fonction harmonique de pulsation $\omega$ non nulle, c'est-à-dire $u(x, t)=u(x) e^{i \omega t}$ (ce qui correspond à une transformation de Fourier par rapport à la variable temps $t$ ), alors l'équation (1.2) nous conduit à la résolution de

$$
\forall x \in \mathbb{R}_{+}^{n}, \quad\left(\Delta_{e}-\omega^{2}\right) u(x)=F_{\omega}(x)
$$

où $F_{\omega}$ est la transformée de Fourier, par rapport à $t$, de la fonction $F$. En fait la résolution de l'équation (1.3) se ramène à la recherche d'une solution $G(x, y ; \omega)$ de

$$
\forall x \in \mathbb{R}_{+}^{n} \quad\left(\Delta_{e}-\omega^{2}\right) u(x)=\delta_{y}(x)
$$

où $\delta$ est la mesure de Dirac en $y$ (point source), avec $G$ vérifiant les conditions imposées sur le bord de $\Omega$. Dans ce cas,

$$
u(x)=\int_{\mathbb{R}_{+}^{n}} G(x, y ; \omega) F_{\omega}(y) \mathrm{d} y
$$

est solution de l'équation (1.3) dans $\mathbf{H}$. 


\section{Philippe Sécher}

Calculons $G$. On commence par chercher les solutions

$$
u: \mathbb{R}_{+}^{n} \rightarrow \mathbb{R}^{n} \text { de }\left(\Delta_{e}-\omega^{2}\right) u=0 .
$$

C'est à partir de ces solutions, appelées fonctions propres ou fonctions propres généralisées, que, par superposition, l'on construit $G(x, y ; \omega)$.

Commençons par décrire les ondes planes intervenant dans $\mathbb{R}^{n}$. Dans la suite on les qualifiera d'ondes incidentes. On considère $u$ une onde plane (vectorielle) de vecteur d'onde $\xi$ de pulsation $\omega$, c'est-à-dire $u(x, t)=$ $d e^{i(\langle x, \xi\rangle+\omega t)}$ avec

$$
\langle x, \xi\rangle=\sum_{k=1}^{n} x_{k} \xi_{k} \quad \text { et } \quad \xi \in \mathbb{R}^{n} \text {. }
$$

$u$ est solution non triviale de $\left(\Delta_{e}+\partial^{2} / \partial t^{2}\right) u=0$ équivaut à $d$ solution non nulle de l'équation algébrique suivante :

$$
\Delta_{e}(\xi) d=\omega^{2} d
$$

avec

$$
\Delta_{e}(\xi)=\frac{\mu}{\rho}|\xi|^{2} I_{n}+\frac{\lambda+\mu}{\rho} M_{n}
$$

où $M_{n}$ est la matrice $n \times n$ de coefficients $\left(M_{n}\right)_{j, k}=\xi_{j} \xi_{k}$ et $|\xi|^{2}=\sum_{k=1}^{n} \xi_{k}^{2}$. $d$ différent de zéro entraîne la formulation de l'équation spectrale suivante entre $\omega$ et $\xi$,

$$
D(\xi, \omega) \equiv \operatorname{det}\left(\Delta_{e}(\xi)-\omega^{2} I\right)=0 .
$$

Ce déterminant vaut

$$
D(\xi, \omega)=\left(\frac{\mu}{\rho}|\xi|^{2}-\omega^{2}\right)^{n-1}\left(\frac{\lambda+2 \mu}{\rho}|\xi|^{2}-\omega^{2}\right) .
$$

L'équation $D(\xi, \omega)=0$ conduit à deux relations entre $\xi$ et $\omega$ donc à deux types d'ondes :

- onde longitudinale $\omega^{2}=c_{L}^{2}|\xi|^{2}$ avec

$$
c_{L}=\sqrt{\frac{\lambda+2 \mu}{\rho}} ;
$$


- onde transversale $\omega^{2}=c_{T}^{2}|\xi|^{2}$ avec

$$
c_{T}=\sqrt{\frac{\mu}{\rho}} .
$$

On a donc décrit les différents types d'ondes incidentes. On retrouve un résultat du même type qu'en dimension trois. Situons sur le dessin ci-dessous les deux vitesses intervenant.

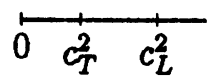

Fig. 1

Revenons à l'expression de $G$. À l'aide des ondes planes incidentes, on construit des lois de réflexion, cela fournit une partie des modes propres liés au problème du demi-espace. Par cette technique on n'obtient pas l'onde de Rayleigh [R], cette onde ne correspond pas à une superposition d'ondes planes. Cette onde est, comme on va le voir plus loin, liée a l'apparition de spectre ponctuel dans le problème à bord libre. Pour obtenir $G$ en s'assurant de la complétude des ondes considérées, on utilise la théorie spectrale des opérateurs autoadjoints. On doit donc faire une étude spectrale de l'opérateur $\Delta_{e}$ d'élasticité linéaire de paramètre spectral $\omega^{2}$. Pour l'étude spectrale on sait, voir [Sh], qu'il suffit de considérer des fonctions propres généralisées bornées. Sur $\mathbb{R}_{+}^{n}$, on considère donc les ondes $u(x, t)=d e^{i(\langle x, \xi\rangle+\omega t)}$ avec $\xi^{\prime} \equiv\left(\xi_{2}, \ldots, \xi_{n}\right)$ dans $\mathbb{R}^{n-1}$ et $\xi_{1}$ complexe à partie imaginaire positive.

Pour effectuer la décomposition spectrale, on construit une transformation unitaire qui diagonalise l'opérateur $\Delta_{e}$. Aussi on commence par le découpler autant que possible.

On utilise ici la même technique que Wilcox [W]. On commence par effectuer une transformation de Fourier partielle par rapport aux variables $x_{2}, x_{3}, \ldots, x_{n}$.

Ensuite, la symétrie cylindrique du problème par rapport à l'axe des $x_{1}$ nous conduit à effectuer une rotation $U$ autour de cet axe afin de se ramener dans un repère pour lequel $\xi^{\prime}=\left(\left|\xi^{\prime}\right|, 0\right)$ où $\left|\xi^{\prime}\right|=\left(\xi_{2}^{2}+\cdots+\xi_{n}^{2}\right)^{1 / 2}$.

Effectuons alors le changement de coordonnées $x_{1}^{\prime}=\left|\xi^{\prime}\right| x_{1}$. Dans ce nouveau système de coordonnées,

$$
\begin{gathered}
\frac{\partial}{\partial x_{1}}=\left|\xi^{\prime}\right| \frac{\partial}{\partial x_{1}^{\prime}} \\
-703-
\end{gathered}
$$


et l'opérateur d'élasticité linéaire s'écrit

$$
\Delta_{e}^{\left|\xi^{\prime}\right|}=\frac{\mu}{\rho}\left|\xi^{\prime}\right|^{2}\left(\begin{array}{ccc}
-\frac{\lambda+2 \mu}{\mu} \frac{\partial^{2}}{\partial x_{1}^{\prime 2}}+1 & -i \frac{\lambda+\mu}{\mu} \frac{\partial}{\partial x_{1}^{\prime}} & \\
-i \frac{\lambda+\mu}{\mu} \frac{\partial}{\partial x_{1}^{\prime}} & \frac{\lambda+2 \mu}{\mu}-\frac{\partial^{2}}{\partial x_{1}^{\prime 2}} & \\
& 0 & \\
& & \left(1-\frac{\partial^{2}}{\partial x_{1}^{\prime 2}}\right) I_{n-2}
\end{array}\right)
$$

d'où

$$
\Delta_{e}^{\left|\xi^{\prime}\right|}=\frac{\mu}{\rho}\left|\xi^{\prime}\right|^{2}\left(A \oplus(-\Delta+1) I_{n-2}\right)
$$

oú $A$ est l'opérateur défini par la formule (0.1) avec $c^{2}=(\lambda+2 \mu) / \mu>1$ et $\Delta$ est le Laplacien.

Regardons maintenant comment les différentes transformations unitaires effectuées sur $\Delta_{e}$ transforment la condition au bord. Après transformation, la condition de bord libre conduit $v={ }^{t}\left(v_{1}, v_{2}, \ldots, v_{n}\right)$ à vérifier

$$
\left(\begin{array}{l}
v_{1} \\
v_{2}
\end{array}\right) \in \mathcal{D} \quad \text { et }\left.\quad \frac{\partial v_{j}}{\partial x_{1}^{\prime}}\right|_{x_{1}^{\prime}=0}=0 \quad \text { pour } 3 \leq j \leq n .
$$

Le découplage observé au niveau de l'opérateur reste vrai au niveau des conditions au bord.

En conclusion on a un découplage de l'opérateur. L'étude de $\Delta_{e}$ sur $L^{2}\left(\mathbb{R}_{+}^{n}\right)$ avec des conditions de bord libre se déduit donc de celle de $-\Delta$ sur $L^{2}\left(\mathbb{R}_{+}\right)$avec condition de Neumann et de celle de $A$ sur $\mathcal{D}$. L'étude de $-\Delta$ muni des conditions de Neumann étant classique, on étudie seulement $A, \mathcal{D}$.

Avant d'entamer l'étude de $A, \mathcal{D}$ définissons les notations que l'on utilisera par la suite. Dans ce qui suit on notera :

$$
\begin{gathered}
u=\left(\begin{array}{c}
u_{1} \\
u_{2}
\end{array}\right) \quad \bar{u}=\left(\overline{u_{1}}, \overline{u_{2}}\right) \\
\int \overline{u(x)} u(x) \mathrm{d} x=\int\left(\left|u_{1}(x)\right|^{2}+\left|u_{2}(x)\right|^{2}\right) \mathrm{d} x
\end{gathered}
$$

et

$$
\int u(x) \overline{u(x)} \mathrm{d} x=\left(\begin{array}{cc}
\int\left|u_{1}(x)\right|^{2} \mathrm{~d} x & \int u_{2}(x) \overline{u_{1}(x)} \mathrm{d} x \\
\int u_{1}(x) \overline{u_{2}(x)} \mathrm{d} x & \int\left|u_{2}(x)\right|^{2} \mathrm{~d} x
\end{array}\right) .
$$


Étude spectrale du système différentiel $2 \times 2$ associé à un problème d'élasticité linéaire Définissons pour $z \in \mathbb{C} \backslash \mathbb{R}^{+}$,

$$
T(z)=(z-1)^{1 / 2} \text { et } L(z)=\left(\frac{z}{c^{2}}-1\right)^{1 / 2}
$$

où pour détermination de la racine carrée complexe on choisit la définition suivante.

DÉfinition. - Pour tout $z$ complexe on définit $(z)^{1 / 2}$ par

$$
\begin{cases}(z)^{1 / 2}=\sqrt{z} & \text { si } z \in \mathbb{R}^{+} \\ (z)^{1 / 2}=\sqrt{r} e^{i \theta / 2} & \text { si } \left.z \in \mathbb{C} \backslash \mathbb{R}^{+}, z=r e^{i \theta} \text { avec } r \geq 0 \text { et } \theta \in\right] 0,2 \pi[.\end{cases}
$$

À partir de la définition de $T$ et $L$ pour tout $z$ dans $\mathbb{C} \backslash \mathbb{R}^{+}$, on définit $T$ et $L$ pour $z=k$ réel positif à l'aide du principe de limite d'absorption en considérant :

$T(k)=\lim _{\gamma \rightarrow 0_{+}} T(k+i \gamma) \equiv T(k+i 0) \quad$ et $\quad L(k)=\lim _{\gamma \rightarrow 0_{+}} L(k+i \gamma) \equiv L(k+i 0)$.

$\grave{A}$ partir de cela, on définit, pour tout $z$ dans $\mathbb{C}$,

$$
\begin{aligned}
& u_{T}^{ \pm}(x, z)=\left(\begin{array}{c}
1 \\
\mp T(z)
\end{array}\right) e^{ \pm i x T(z)} \\
& u_{L}^{ \pm}(x, z)=\left(\begin{array}{c} 
\pm L(z) \\
1
\end{array}\right) e^{ \pm i x L(z)} .
\end{aligned}
$$

Remarque. - Pour tout $z, u_{T}^{ \pm}(\cdot, z)$ et $u_{L}^{ \pm}(\cdot, z)$ vérifient $(A-z) u=0$.

Par les notations adoptées pour $T, L, u_{T}^{ \pm}, u_{L}^{ \pm}$et la racine carrée complexe, on a de façon évidente les relations suivantes.

Proposition 1.1. - Si $k \in \mathbb{C} \backslash \mathbb{R}^{+}$,

$$
\begin{aligned}
& T(k+i 0)=T(k-i 0), \quad L(k+i 0)=L(k-i 0) \\
& u_{T}^{ \pm}(x, k+i 0)=u_{T}^{ \pm}(x, k-i 0), \quad u_{L}^{ \pm}(x, k+i 0)=u_{L}^{ \pm}(x, k-i 0) .
\end{aligned}
$$

Si $k \in\left[c^{2}, \infty[\right.$,

$$
\begin{gathered}
T(k+i 0)=-T(k-i 0), \quad L(k+i 0)=-L(k-i 0) \\
u_{T}^{ \pm}(x, k+i 0)=u \mp(x, k-i 0), \quad u_{L}^{ \pm}(x, k+i 0)=u_{L}^{\mp}(x, k-i 0) . \\
-705-
\end{gathered}
$$


Si $k \in\left[1, c^{2}[\right.$,

$$
\begin{aligned}
& T(k+i 0)=-T(k-i 0), \quad L(k+i 0)=L(k-i 0) \\
& u_{T}^{ \pm}(x, k+i 0)=u \varsubsetneqq(x, k-i 0), \quad u_{L}^{ \pm}(x, k+i 0)=u_{L}^{ \pm}(x, k-i 0) .
\end{aligned}
$$

Si $k \in[0,1[$,

$$
\begin{aligned}
& T(k+i 0)=T(k-i 0), \quad L(k+i 0)=L(k-i 0) \\
& u_{T}^{ \pm}(x, k+i 0)=u_{T}^{ \pm}(x, k-i 0), \quad u_{L}^{ \pm}(x, k+i 0)=u_{L}^{ \pm}(x, k-i 0) .
\end{aligned}
$$

Maintenant passons à la construction des fonctions propres généralisées à partir du calcul des lois de réflexion.

\section{Calcul des fonctions propres généralisées}

Le noyau de Green $G(x, y ; z)$ associé à $A-z$ sur le domaine $\mathcal{D}$ vérifie

$$
(A-z) G(x, y ; z)=\left(\begin{array}{cc}
\delta_{y}(x) & 0 \\
0 & \delta_{y}(x)
\end{array}\right) \quad \text { et }\left.\quad a(G)\right|_{x=0}=\left.b(G)\right|_{x=0}=0 .
$$

On va chercher $G$ sous la forme d'une superposition d'un terme incident $G^{i}$, qui correspond au noyau de Green pour le domaine entier $H^{2}(\mathbb{R})$, et d'un terme réfléchi $G^{r}$.

On écrit que $\forall z \in \mathbb{C} \backslash \mathbb{R}^{+}, \forall x, y \in \mathbb{R}^{+}$,

$$
G(x, y ; z)=G^{i}(x, y ; z)+G^{r}(x, y ; z) .
$$

Dans ce cas, $G^{r}$ doit vérifier

$$
(A-z) G^{r}(x, y ; z)=0
$$

et

$$
\left\{\begin{array}{l}
\left.a\left(G^{r}\right)\right|_{x=0}=-\left.a\left(G^{i}\right)\right|_{x=0} \\
\left.b\left(G^{r}\right)\right|_{x=0}=-\left.b\left(G^{i}\right)\right|_{x=0}
\end{array}\right.
$$

On calcule d'abord $G^{r}(x, y ; z)$ pour $y>x$, qui doit vérifier la condition au bord en $x=0$. Pour calculer $G^{r}$ à partir de $G^{i}$, établissons d'abord 
Étude spectrale du système différentiel $2 \times 2$ associé à un problème d'élasticité linéaire ce que l'on peut appeler des lois de réflexion, liées aux ondes solutions de $(A-z) u=0$ avec $z \in \mathbb{C} \backslash \mathbb{R}^{+}$.

Soit $z \in \mathbb{C} \backslash \mathbb{R}^{+}:$les quatre ondes $u_{T}^{ \pm}(\cdot, z)$ et $u_{L}^{ \pm}(\cdot, z)$ sont alors indépendantes, $u_{T}^{+}(\cdot, z), u_{L}^{+}(\cdot, z)$ sont bornées sur $\mathbb{R}^{+}$et $u_{T}^{-}(\cdot, z), u_{L}^{-}(\cdot, z)$ le sont sur $\mathbb{R}^{-}$.

À partir de cela donnons quelques définitions.

DÉfINITION. - On note

$$
\begin{aligned}
& E^{+}(z) \text { l'espace engendré par } u_{T}^{+}(\cdot, z) \text { et } u_{L}^{+}(\cdot, z) \\
& \Phi^{+}(z): E^{+}(z) \longrightarrow \mathbb{C}^{2} \\
& u \longmapsto\left(\left.a(u)\right|_{x=0},\left.b(u)\right|_{x=0}\right) .
\end{aligned}
$$

$E^{+}$est l'ensemble des solutions bornées sur $\mathbb{R}^{+}$.

Par définition de $u_{T}^{ \pm}$et $u_{L}^{ \pm}$, on a

$$
\left\{\begin{array} { l } 
{ a ( u _ { L } ^ { + } ) = 2 L ( z ) e ^ { i x L ( z ) } } \\
{ b ( u _ { L } ^ { + } ) = ( z - 2 ) e ^ { i x L ( z ) } }
\end{array} \quad \text { et } \quad \left\{\begin{array}{l}
a\left(u_{T}^{+}\right)=(-z+2) e^{i x T(z)} \\
b\left(u_{T}^{+}\right)=2 T(z) e^{i x T(z)}
\end{array}\right.\right.
$$

Le déterminant de $\Phi^{+}(z)$, que l'on note $-D$, vaut donc

$$
-D(z) \equiv\left|\begin{array}{cc}
-z+2 & 2 T(z) \\
2 L(z) & z-2
\end{array}\right|
$$

L'égalité $D(z)=0$ correspond à l'équation

$$
(z-2)^{2}+4 T(z) L(z)=0
$$

qui ne possède pas de racine dans $\mathbb{C} \backslash \mathbb{R}^{+}$(voir par exemple le livre [A]) donc $\Phi^{+}(z)$ est un isomorphisme pour $z \in \mathbb{C} \backslash \mathbb{R}^{+}$.

\section{La condition de Lopatinski est vérifiée}

On a donc deux types de solutions vérifiant les conditions aux limites en $x=0 .\left(\Phi^{+}\right)^{-1}(z)$ est tel que

$$
\begin{aligned}
&\left(\Phi^{+}\right)^{-1}(z): \mathbb{C}^{2} \longrightarrow E^{+}(z) \\
&(a, b) \longmapsto-\frac{a(z-2)-2 b L(z)}{D(z)} u_{T}^{+}+\frac{2 a T(z)+b(z-2)}{D(z)} u_{L}^{+} \\
&-707-
\end{aligned}
$$




\section{Philippe Sécher}

Pour cette construction de $\Phi^{+}, u_{T}^{+}$et $u_{L}^{+}$apparaissent comme des "termes réfléchis".

Pour construire le terme réfléchi $G^{r}$ tel que $\left.a(G)\right|_{x=0}=\left.b(G)\right|_{x=0}=0$, on part de $G^{i},\left.a\left(G^{i}\right)\right|_{x=0}$ et $\left.b\left(G^{i}\right)\right|_{x=0}$.

Comme on considère la trace pour $x=0$ on s'intéresse à l'expression de $G^{i}$ pour $y>x$. On a dans ce cas, en exprimant $G^{i}$ à l'aide de $u_{T}^{\bar{T}}$ et $u_{\bar{L}}$ (voir par exemple [Se]),

$$
G^{i}(x, y ; z)=\frac{i}{2 z}\left[\frac{u_{T}^{-}(x, z)^{t} u_{\bar{T}}(-y, z)}{T(z)}+\frac{u_{L}^{-}(x, z)^{t} u_{L}^{-}(-y, z)}{L(z)}\right] .
$$

Les termes associés à $u_{\bar{L}}^{-}$et $u_{T}^{-}$conduisent à des valeurs de $a$ et $b$ non nulles sur le bord, on doit donc associer à chaque terme, par $\left(\Phi^{+}\right)^{-1}$, une partie "réfléchie".

- Si on considère l'onde $u_{T}^{-}(x, z)$, elle conduit à

$$
a\left(u_{T}^{\bar{T}}\right)=(-z+2) e^{-i x T(z)} \quad \text { et } \quad b\left(u_{T}^{\bar{T}}\right)=-2 T(z) e^{-i x T(z)} .
$$

L'onde "réfléchie" associée à $u_{T}^{-}(x, z)$ vaut dans ce cas $\Phi_{+}^{-1}(z-2 ; 2 T(z))$, c'est-à-dire

$$
-\frac{4 L(z) T(z)-(z-2)^{2}}{D(z)} u_{T}^{+}(x, z)+\frac{4 T(z)(z-2)}{D(z)} u_{L}^{+}(x, z) .
$$

- Si on considère maintenant l'onde "incidente" $u_{\bar{L}}^{-}(x, z)$,

$$
a\left(u_{\bar{L}}\right)=-2 L(z) e^{-i x L(z)} \quad \text { et } \quad b\left(u_{L}^{\bar{L}}\right)=(z-2) e^{-i x L(z)} .
$$

L'onde "réfléchie" vaut dans ce cas :

$$
-\frac{4 L(z)(z-2)}{D(z)} u_{T}^{+}(x, z)+\frac{4 L(z) T(z)-(z-2)^{2}}{D(z)} u_{L}^{+}(x, z) .
$$

À partir de ces "lois de réflexion", on définit les fonctions suivantes :

$$
\begin{gathered}
\phi_{1}(x, z)=\left[u_{T}^{-}+R_{2} u_{T}^{+}+T R_{1} u_{L}^{+}\right](x, z) \\
\phi_{2}(x, z)=\left[u_{L}^{-}-L R_{1} u_{T}^{+}+R_{2} u_{L}^{+}\right](x, z) \\
-708-
\end{gathered}
$$


Étude spectrale du système différentiel $2 \times 2$ associé à un problème d'élasticité linéaire où

$$
\begin{aligned}
& R_{1}(z)=\frac{4(z-2)}{(z-2)^{2}+4 T(z) L(z)} \\
& R_{2}(z)=\frac{4 T(z) L(z)-(z-2)^{2}}{(z-2)^{2}+4 T(z) L(z)} .
\end{aligned}
$$

\section{Remarques}

- Les deux coefficients $R_{1}, R_{2}$ sont liés par la relation $R_{1}^{2} L T+R_{2}^{2}=1$.

- Les lois de réflexion, pour $z$ dans $\mathbb{C} \backslash \mathbb{R}^{+}$, sont du type

$$
\left(\frac{u_{T}^{-}}{T}, \frac{u_{L}^{-}}{L}\right) \rightarrow\left(\frac{u_{T}^{+}}{T}, \frac{u_{L}^{+}}{L}\right) r_{S} \quad \text { où } r_{S}=\left(\begin{array}{cc}
R_{2} & -T R_{1} \\
L R_{1} & R_{2}
\end{array}\right) \text {. }
$$

Revenons maintenant au calcul de $G^{r}$. À l'aide des lois de réflexion, on construit $G^{r}(x, y ; z)$, pour $z \in \mathbb{C} \backslash \mathbb{R}^{+}$dans le cas $y>x$,

$$
\begin{aligned}
G^{r}(x, y ; z)= & \frac{i}{2 z} \frac{u_{T}^{+}(x, z)}{T(z)}\left(R_{2}{ }^{t} u_{T}^{-}-T R_{1}{ }^{t} u_{L}^{-}\right)(-y, z) \\
& +\frac{i}{2 z} \frac{u_{L}^{+}(x, z)}{L(z)}\left(L R_{1}{ }^{t} u_{T}^{-}+R_{2}{ }^{t} u_{\bar{L}}^{-}\right)(-y, z) .
\end{aligned}
$$

Par construction $G^{r}$ vérifie

$$
(A-z) G^{r}=0 \quad \text { et } \quad\left\{\begin{array}{l}
\left.a\left(G^{r}\right)\right|_{x=0}=-\left.a\left(G^{i}\right)\right|_{x=0} \\
\left.b\left(G^{r}\right)\right|_{x=0}=-\left.b\left(G^{i}\right)\right|_{x=0}
\end{array}\right.
$$

ainsi $G^{r}$ n'a pas de singularité, elle est continue.

On va maintenant construire $G^{r}$ pour $y \leq x$. Dans ce domaine $G^{r}$ est bornée si elle est du type

$$
G^{r}(x, y ; z)=u_{T}^{+}(x, z) \alpha_{1}(y)+u_{L}^{+}(x, z) \alpha_{2}(y) .
$$

Donc, la continuité sur la diagonale $x=y$ conduit pour tout $x$ dans $\mathbb{R}^{+} \grave{\mathrm{a}}$

$$
\begin{aligned}
& u_{T}^{+}(x, z) \alpha_{1}(x)+u_{L}^{+}(x, z) \alpha_{2}(x)= \\
& =\frac{i}{2 z^{2}}\left[\frac{u_{T}^{+}(x, z)}{T(z)}\left(R_{2}{ }^{t} u_{T}^{-}-T R_{1}{ }^{t} u_{L}^{-}\right)(-x, z)\right. \\
& \left.\quad+\frac{u_{L}^{+}(x, z)}{L(z)}\left(L R_{1}{ }^{t} u_{T}^{-}+R_{2}{ }^{t} u_{\bar{L}}^{-}\right)(-x, z)\right] .
\end{aligned}
$$


Mais, pour $z \in \mathbb{C} \backslash \mathbb{R}^{+}, u_{T}^{+}(x, z)$ et $u_{L}^{+}(x, z)$ sont linéairement indépendantes d'où

$$
\begin{aligned}
& \alpha_{1}(x)=\frac{i}{2 z T(z)}\left(R_{2}{ }^{t} u_{T}^{-}-T R_{1}{ }^{t} u_{L}^{-}\right)(-x, z) \\
& \alpha_{2}(x)=\frac{i}{2 z L(z)}\left(L R_{1}{ }^{t} u_{T}^{-}+R_{2}{ }^{t} u_{L}^{-}\right)(-x, z) .
\end{aligned}
$$

Ce qui entraîne, pour tout $x, y$,

$$
\begin{aligned}
G^{r}(x, y ; z)= & \frac{i}{2 z} \frac{u_{T}^{+}(x, z)}{T(z)}\left(R_{2}{ }^{t} u_{T}^{-}-T R_{1}{ }^{t} u_{L}^{-}\right)(-y, z) \\
& +\frac{i}{2 z} \frac{u_{L}^{+}(x, z)}{L(z)}\left(L R_{1}{ }^{t} u_{T}^{-}+R_{2}{ }^{t} u_{L}^{\bar{L}}\right)(-y, z)
\end{aligned}
$$

vérifiant, $(A-z) G^{r}=0$. D'où $G=G^{i}+G^{r}$ vérifie bien

$$
(A-z) G(x, y ; z)=\left(\begin{array}{cc}
\delta_{y}(x) & 0 \\
0 & \delta_{y}(x)
\end{array}\right)
$$

et

$$
\left.a(G)\right|_{x=0}=\left.b(G)\right|_{x=0}=0 .
$$

Pour $G$ on a l'expression suivante :

$$
G(x, y ; z)=\frac{i}{2 z} \begin{cases}\frac{\phi_{1}(x, z) \overline{u_{T}^{+}(y, \bar{z})}}{T(z)}+\frac{\phi_{2}(x, z) \overline{u_{L}^{+}(y, \bar{z})}}{L(z)} & \text { si } y>x \\ \frac{u_{T}^{+}(x, z) \overline{\phi_{1}(y, \bar{z})}}{T(z)}+\frac{u_{L}^{+}(x, z) \overline{\phi_{2}(y, \bar{z})}}{L(z)} & \text { si } y<x\end{cases}
$$

et ceci pour tout $z \in \mathbb{C} \backslash \mathbb{R}^{+}$.

$\mathrm{Au}$ cours de la démonstration précédente, on a construit les lois de réflexion, dues à la présence de la surface libre, pour $z$ complexe en relation avec la condition de Lopatinski. Étudions le cas $z=k$ réel positif, en regardant la condition de Lopatinski uniforme. La construction de ces lois de réflexion conduit à l'obtention des fonctions propres généralisées. 
Étude spectrale du système différentiel $2 \times 2$ associé à un problème d'élasticité linéaire

Commençons par définir, pour $z=k$ réel positif, $D(k)$ :

$$
D(k)=D(k+i 0)
$$

puis pour $k$ tel que $D(k) \neq 0$ définissons $R_{1}(k), R_{2}(k), \phi_{1}(k)$ et $\phi_{2}(k)$,

$$
\begin{array}{ll}
R_{1}(k)=R_{1}(k+i 0), & R_{2}(k)=R_{2}(k+i 0) \\
\phi_{1}(k)=\phi_{1}(k+i 0), & \phi_{2}(k)=\phi_{2}(k+i 0) .
\end{array}
$$

\section{Remarques}

- Les expressions de $D, R_{1}, R_{2}, \phi_{1}$ et $\phi_{2}$ restent les mêmes pour $z=k$ réel positif, grâce aux définitions adoptées pour $L(k), T(k), u_{T}^{ \pm}(k)$ et $u_{L}^{ \pm}(k)$.

$$
\begin{gathered}
D(k)=(k-2)^{2}+4 T(k) L(k) \\
R_{1}(k)=\frac{4(k-2)}{(k-2)^{2}+4 T(k) L(k)} \\
R_{2}(k)=\frac{4 T(k) L(k)-(k-2)^{2}}{(k-2)^{2}+4 T(k) L(k)} \\
\phi_{1}(k)=\left[u_{T}^{-}+R_{2} u_{T}^{+}+T R_{1} u_{L}^{+}\right](x, k) \\
\phi_{2}(k)=\left[u_{\bar{L}}^{-}-L R_{1} u_{T}^{+}+R_{2} u_{L}^{+}\right](x, k) .
\end{gathered}
$$

- La relation $R_{1}^{2} L T+R_{2}^{2}=1$ reste vérifiée.

- L'espace $E^{+}(k+i \gamma)$ admet une limite quand $\gamma$ tend vers zéro de manière positive, $u_{T}^{+}(\cdot, z)$ et $u_{L}^{+}(\cdot, z)$ restant bornées sur $\mathbb{R}^{+}$pour $z=k$ dans $\mathbb{R}^{+}$. On pose

$$
E^{+}(k)=E^{+}(k+i 0) \text {. }
$$

C'est l'espace suivant :

$$
E^{+}(k)=\left\{u \mid u=\alpha u_{T}^{+}(k)+\beta u_{L}^{+}(k) \text { avec } \alpha, \beta \in \mathbb{C}^{2} ; k \in \mathbb{R}^{+}\right\} .
$$

Proposition 2.1. - La condition de Lopatinski uniforme n'est pas vérifiée. 
Démonstration. - La condition de Lopatinski uniforme (cf. [CP]) est vérifiée si et seulement si l'application

$$
\begin{aligned}
\Phi^{+}(k): E^{+}(k) & \longrightarrow \mathbb{C}^{2} \\
u & \longmapsto\left(\left.a(u)\right|_{x=0},\left.b(u)\right|_{x=0}\right)
\end{aligned}
$$

est un isomorphisme pour tout $k$ réel positif différent de 0 .

L'application $\Phi^{+}(k)$ est définie par

$$
\Phi^{+}(k)\left(\alpha u_{T}^{+}+\beta u_{L}^{+}\right)=(\alpha, \beta)\left(\begin{array}{cc}
2-k & 2 T(k) \\
2 L(k) & k-2
\end{array}\right) .
$$

On voit que c'est un isomorphisme si

$$
\left|\begin{array}{cc}
-k+2 & 2 T(k) \\
2 L(k) & k-2
\end{array}\right|=-D(k)
$$

est non nul.

L'égalité $D(k)=0$ correspond à l'équation de Rayleigh [R]. On sait (cf. [A]) qu'elle possède deux racines que l'on note 0 et $\omega_{R}^{2}$ avec $\left.\omega_{R} \in\right] 0,1$ [. Pour ces valeurs $\Phi^{+}(k)$ n'est plus un isomorphisme.

La condition de Lopatinski uniforme n'est pas vérifiée (pour $k=\omega_{R}^{2}$ ).

Maintenant intéressons-nous aux lois de réflexion pour $k$ réel positif. Rappelons dans ce cas la notion d'onde incidente.

DÉfinition. - Pour $k$ réel positif fixé, $u_{L}^{-}(x, k)$ et $u_{T}^{-}(x, k)$ sont appelées ondes incidentes quand elles sont bornées sur le domaine d'étude. Sur $\mathbb{R}^{+}, u_{L}^{-}(\cdot, k)$ est bornée si $k$ est dans $\left[c^{2}, \infty\left[\right.\right.$ et $u_{T}^{-}(\cdot, k)$ est bornée si $k$ est dans $[1, \infty[$.

Exprimons les lois de réflexion, pour $k$ réel positif.

\section{THÉORÈME 2.2}

- Soit $k \in\left[c^{2}, \infty[\right.$; pour ces valeurs de $k$ il existe deux types d'ondes incidentes. Ces ondes sont $u_{L}^{-}(k)$ et $u_{T}^{-}(k)$. L'onde réfléchie associée à l'onde incidente $u_{T}^{-}(x, k)$ vaut

$$
\begin{gathered}
-\frac{4 L(k) T(k)-(k-2)^{2}}{D(k)} u_{T}^{+}(x, k)+\frac{4 T(k)(k-2)}{D(k)} u_{L}^{+}(x, k) . \\
-712-
\end{gathered}
$$


Étude spectrale du système différentiel $2 \times 2$ associé à un problème d'élasticité linéaire

L'onde réfléchie associée à l'onde incidente $u_{L}^{-}(x, k)$ vaut

$$
-\frac{4 L(k)(k-2)}{D(k)} u_{T}^{+}(x, k)+\frac{4 L(k) T(k)-(k-2)^{2}}{D(k)} u_{L}^{+}(x, k) .
$$

- Soit $k \in\left[1, c^{2}\left[\right.\right.$; il n'existe alors qu'une onde incidente qui est $u_{T}^{-}(k)$. $L$ 'onde réfléchie associée à l'onde incidente $u_{T}^{-}(x, k)$ vaut

$$
\frac{4 L(k) T(k)-(k-2)^{2}}{D(k)} u_{T}^{+}(x, k)+\frac{4 T(k)(k-2)}{D(k)} u_{L}^{+}(x, k) .
$$

- Soit $k \in[0,1[$; il n'existe alors pas d'ondes incidentes. Mais, pour $k=\omega_{R}^{2}$, définie précédemment, on peut construire une fonction $\phi_{R}(x)$ de $L^{2}\left(\mathbb{R}^{+}\right)$telle que $A \phi_{R}=\omega_{R}^{2} \phi_{R}$ et $\Phi^{+}\left(\omega_{R}^{2}\right) \phi_{R}=0$

$$
\begin{aligned}
\phi_{R}(x)= & \left(2-\omega_{R}^{2}\right)\left(\begin{array}{c}
-\sqrt{1-\omega_{R}^{2} / c^{2}} \\
i
\end{array}\right) e^{-x \sqrt{1-\omega_{R}^{2} / c^{2}}}+ \\
& -\sqrt{1-\frac{\omega_{R}^{2}}{c^{2}}}\left(\begin{array}{c}
-1 \\
i \sqrt{1-\omega_{R}^{2}}
\end{array}\right) e^{-x \sqrt{1-\omega_{R}^{2}}} .
\end{aligned}
$$

Démonstration. - On construit les lois de réflexion de manière à vérifier la condition de contrainte nulle sur le bord. Conformément à ce qui est fait dans le livre de [CP], on part des lois de réflexion obtenues pour $z$ dans $\mathbb{C} \backslash \mathbb{R}^{+}$et on effectue un passage à la limite en ne considérant que les termes bornés. Cela conduit aux trois domaines d'existence pour les ondes incidentes.

Contrairement au cas $z \in \mathbb{C} \backslash \mathbb{R}^{+}, \Phi^{+}(k)$ n'est pas toujours un isomorphisme aussi doit-on étudier son noyau qui est formé d'éléments de $E^{+}(k)$ vérifiant la condition de contrainte nulle au bord.

Pour $k \in \mathbb{R} \backslash\left\{0, \omega_{R}^{2}\right\}, \Phi^{+}(k)$ est un isomorphisme les lois de réflexion obtenues par passage à la limite sont valables, elles conduisent à $\phi_{1}(\cdot, k)$ et $\phi_{2}(\cdot, k)$.

Pour $k=\omega_{R}^{2}, \Phi^{+}(k)$ n'est pas un isomorphisme. Son noyau est de dimension un, car $\Phi^{+}(k)$ est non nulle, il contient une fonction $\phi_{R}$ définie par

$$
\begin{gathered}
\phi_{R}(x)=\left.\frac{D(z) \phi_{1}(x, z)}{4 i T(z)}\right|_{z=\omega_{R}^{2}}=\left.\frac{D(z) \phi_{2}(x, z)}{2 i(2-z)}\right|_{z=\omega_{R}^{2}} . \\
-713-
\end{gathered}
$$




\section{Philippe Sécher}

Pour $k=0, \Phi^{+}(k)$ est une application non nulle de $E^{+}(0)$ dans $\mathbb{C}^{2}$. Son noyau est réduit à zéro car l'espace de départ est de dimension 1 , en effet, $u_{L}^{+}(0)=i u_{T}^{+}(0)$.

\section{Remarques}

- Par ce choix de l'isomorphisme $\Phi^{+}$, on retrouve les lois classiquement énoncées dans les ouvrages de physique traitant de ce problème, comme par exemple ceux de $[\mathrm{A}],[\mathrm{B}]$ et [EJP], etc.

- On remarque que le spectre ponctuel de $A, \mathcal{D}$ est seulement composé de $\omega_{R}^{2}$.

À partir de ces lois de réflexions on définit les fonctions propres généralisées suivantes.

DÉfInITION. - Soient

$$
\begin{aligned}
\varphi_{R}(x) & =\frac{\phi_{R}(x)}{\left\|\phi_{R}\right\|} \\
\varphi_{1}(x, k) & =\frac{1}{2(\pi T(k) k)^{1 / 2}} \phi_{1}(x, k) \\
\varphi_{2}(x, k) & =\frac{1}{2(\pi L(k) k)^{1 / 2}} \phi_{2}(x, k) .
\end{aligned}
$$

Maintenant, à partir de celles-ci on va construire une transformation unitaire $\Phi$ qui diagonalise $A$, c'est-à-dire telle que $\Phi A \Phi^{*}$ soit un opérateur de multiplication. On obtiendra ainsi la résolution spectrale de $A, \mathcal{D}$.

\section{Résolution spectrale de $A, \mathcal{D}$}

Contrairement au cas de l'espace entier, on ne connait pas a priori la transformation unitaire à considérer pour diagonaliser $A, \mathcal{D}$. On commence par considérer les transformations de Fourier associées aux fonctions propres généralisées.

DÉfinition. - Pour $f \in L^{2}\left(\mathbb{R}^{+}\right)$, on définit

$$
\begin{aligned}
\Phi_{1}: L^{2}\left(\mathbb{R}^{+}\right) & \longrightarrow L^{2}(] c^{2}, \infty[) \\
f & \longmapsto \tilde{f}_{1}(k)=\lim _{M \rightarrow \infty} \int_{0}^{M} \overline{\varphi_{1}(y, k)} f(y) \mathrm{d} y, \\
& -714-
\end{aligned}
$$


Étude spectrale du système différentiel $2 \times 2$ associé à un problème d'élasticité linéaire

$$
\begin{aligned}
& \Phi_{2}: L^{2}\left(\mathbb{R}^{+}\right) \longrightarrow L^{2}(] c^{2}, \infty[) \\
& f \longmapsto \tilde{f}_{2}(k)=\lim _{M \rightarrow \infty} \int_{0}^{M} \overline{\varphi_{2}(y, k)} f(y) \mathrm{d} y, \\
& \Phi_{1}^{\circ}: L^{2}\left(\mathbb{R}^{+}\right) \longrightarrow L^{2}(] 1, c^{2}[) \\
& f \longmapsto \tilde{f}_{1}^{\circ}(k)=\lim _{M \rightarrow \infty} \int_{0}^{M} \overline{\varphi_{1}(y, k)} f(y) \mathrm{d} y, \\
& \Phi_{R}: L\left(\mathbb{R}^{+}\right) \longrightarrow \mathbb{C} \\
& f \\
& \qquad \tilde{f}_{R}=\int_{0}^{\infty} \overline{\varphi_{R}(y)} f(y) \mathrm{d} y .
\end{aligned}
$$

À partir de celles-ci, on définit une application notée $\Phi$ comme suit.

DÉFINITION. - Soit

$$
\begin{aligned}
\Phi: L^{2}\left(\mathbb{R}^{+}\right) & \longrightarrow L^{2}(] c^{2}, \infty[) \oplus L^{2}(] c^{2}, \infty[) \oplus L^{2}(] 1, c^{2}[) \oplus \mathbb{C} \\
f & \longrightarrow\left(\Phi_{1}(f), \Phi_{2}(f), \Phi_{1}^{\circ}(f), \Phi_{R}(f)\right) .
\end{aligned}
$$

On veut montrer que $\Phi$ est unitaire et diagonalise $A$.

Par définition des $\Phi_{i}$, on a $\forall f \in \mathcal{D}, \Phi_{i}(A f)(k)=k \Phi_{i}(f)(k)$. Il reste à montrer que $\Phi$ est unitaire.

Avant d'étudier le caractère unitaire de $\Phi$ décomposons tout élément $f$ de $L^{2}\left(\mathbb{R}^{+}\right)$à l'aide des modes propres.

THÉORÈME 3.1. - Pour tout $f$ dans $L^{2}\left(\mathbb{R}^{+}\right)$, on a

$$
\begin{aligned}
f(x)= & \int_{0}^{\infty} \varphi_{R}(x) \overline{\varphi_{R}(y)} f(y) \mathrm{d} y+\int_{1}^{\infty} \int_{0}^{\infty} \varphi_{1}(x, k) \overline{\varphi_{1}(y, k)} f(y) \mathrm{d} y \mathrm{~d} k+ \\
& +\int_{c^{2}}^{\infty} \int_{0}^{\infty} \varphi_{2}(x, k) \overline{\varphi_{2}(y, k)} f(y) \mathrm{d} y \mathrm{~d} k .
\end{aligned}
$$

Démonstration. - Pour démontrer cette égalité, on suit la même démarche que dans [BS]. On part de $G$ défini en dehors de l'axe réel.

Comme on l'a signalé précédemment l'opérateur $A, \mathcal{D}$ est autoadjoint positif; son spectre est donc inclus dans $[0, \infty[$. 
Notons $R_{z}=(A-z)^{-1}$ pour tout $z$ dans $\mathbb{C} \backslash[0, \infty[$. Pour un contour $\Gamma_{\delta}$ choisi comme sur la figure 2 , entourant le spectre de $A, \mathcal{D}$, on peut donc écrire le résultat suivant :

$$
\forall f \in L^{2}\left(\mathbb{R}^{+}\right), \quad \frac{i}{2 \pi} \int_{\Gamma_{\delta}} R_{z}(f) \mathrm{d} z=f .
$$

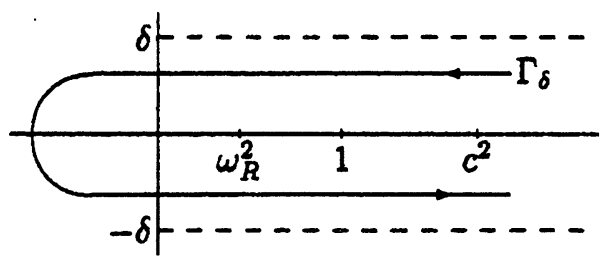

Fig. 2

Ceci correspond à la formule de Stone dans laquelle on considère la résolution spectrale sur tout le spectre. Or

$$
R_{z} f(x)=\int_{0}^{\infty} G(x, y ; z) f(y) \mathrm{d} y, \quad \forall x \geq 0, \forall f \in L^{2}\left(\mathbb{R}^{+}\right)
$$

où $G$ est la fonction de Green définie précédemment.

On a exprimé $G(x, y ; z)$ pour $z \in \mathbb{C} \backslash \mathbb{R}^{+}$mais on peut étendre cette définition à l'ensemble des $z$ vérifiant $D(z) \neq 0$, c'est-à-dire,

$$
\mathbb{C} \backslash\left(\left[1, \infty\left[\cup\left\{0, \omega_{R}^{2}\right\}\right) .\right.\right.
$$

$G$ étant définie à partir de $L(z)$ et de $T(z)$, qui sont deux fonctions holomorphes sur $\mathbb{C}$ privé respectivement de $\left[c^{2}, \infty[\right.$ et de $[1, \infty[$, on en déduit que l'application $z \rightarrow R_{z}$ est holomorphe sur $\mathbb{C} \backslash\left(\left[1, \infty\left[\cup\left\{0, \omega_{R}^{2}\right\}\right)\right.\right.$. D'où par déformation du contour $\Gamma_{\delta}$ en utilisant la propriété d'holomorphie de $G$ on obtient que pour tout $f$ dans $L^{2}\left(\mathbb{R}^{+}\right)$:

$$
\int_{\Gamma_{\delta}} R_{z}(f) \mathrm{d} z=\int_{\Pi_{\delta} \cup \Gamma} R_{z}(f) \mathrm{d} z
$$

où les contours $\Pi_{\delta}$ et $\Gamma$ sont définis sur la figure 3 .

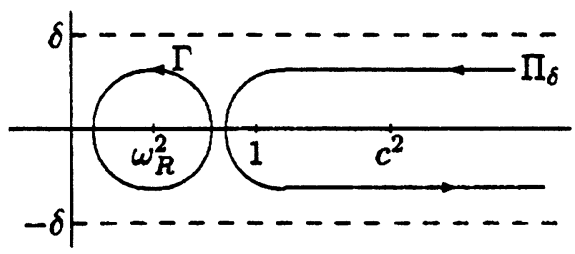

Fig. 3 
Étude spectrale du système différentiel $2 \times 2$ associé à un problème d'élasticité linéaire

On peut isoler les pôles de $G$.

Calculons d'abord l'intégrale sur le contour $\Gamma$.

$$
\begin{aligned}
\int_{\Gamma} R_{z}(f) \mathrm{d} z & =\int_{\Gamma} \int_{0}^{\infty} G(x, y ; z) f(y) \mathrm{d} y \mathrm{~d} z \\
& =\int_{0}^{\infty} 2 i \pi \operatorname{Res}_{\omega_{R}^{2}} G(x, y ; z) f(y) \mathrm{d} y .
\end{aligned}
$$

0 n'est pas un pôle de $G$ car c'est une racine du numérateur du même ordre.

$$
\int_{\Gamma} R_{z}(f) \mathrm{d} z=\left.\int_{0}^{\infty} 2 i \pi \operatorname{Res}_{\omega_{R}^{2}}\left(\frac{1}{D(z)}\right)(D(z) G(x, y ; z))\right|_{z=\omega_{R}^{2}} f(y) \mathrm{d} y .
$$

On montre par le calcul que

$$
\begin{aligned}
\operatorname{Res}_{\omega_{R}^{2}}\left(\frac{1}{D(z)}\right) & =\frac{\left(\omega_{R}^{2}-c^{2}\right)\left(\omega_{R}^{2}-1\right)\left(2-\omega_{R}^{2}\right)}{2 T\left(\omega_{R}^{2}\right) L\left(\omega_{R}^{2}\right)\left[2\left(c^{2}-1\right)+\omega_{R}^{2}\left(1-3 c^{2}\right)+2 \omega_{R}^{4}\right]} \\
\left.D(z) G(x, y ; z)\right|_{z=\omega_{R}^{2}} & =\frac{i}{2 \omega_{R}^{2}}\left(4 i \phi_{R}(x) \overline{u_{T}^{+}\left(y, \omega_{R}^{2}\right)}+\frac{2 i\left(2-\omega_{R}^{2}\right)}{L\left(\omega_{R}^{2}\right)} \phi_{R}(x) \overline{u_{L}^{+}\left(y, \omega_{R}^{2}\right)}\right) \\
& =\frac{1}{\omega_{R}^{2} L\left(\omega_{R}^{2}\right)} \phi_{R}(x) \overline{2 L\left(\omega_{R}^{2}\right) u_{T}^{+}\left(y, \omega_{R}^{2}\right)-\left(2-\omega_{R}^{2}\right) u_{L}^{+}\left(y, \omega_{R}^{2}\right)} \\
& =\frac{1}{i \omega_{R}^{2} L\left(\omega_{R}^{2}\right)} \phi_{R}(x) \overline{\phi_{R}(y)}
\end{aligned}
$$

et

$$
\begin{aligned}
& \int_{\mathbb{R}^{+}} \overline{\phi_{R}(s)} \phi_{R}(s) \mathrm{d} s= \\
& \quad=\left\|\phi_{R}\right\|^{2}=-2 i \omega_{R}^{2} T\left(\omega_{R}^{2}\right) L^{2}\left(\omega_{R}^{2}\right) \frac{2\left(c^{2}-1\right)+\omega_{R}^{2}\left(1-3 c^{2}\right)+2 \omega_{R}^{4}}{\left(\omega_{R}^{2}-c^{2}\right)\left(\omega_{R}^{2}-1\right)\left(2-\omega_{R}^{2}\right)}
\end{aligned}
$$

ce qui conduit à

$$
\int_{\Gamma} R_{z}(f) \mathrm{d} z=-\int_{0}^{\infty} 2 i \pi \frac{\phi_{R}(x) \overline{\phi_{R}(y)}}{\left\|\phi_{R}\right\|^{2}} f(y) \mathrm{d} y
$$

d'où

$$
\begin{aligned}
\frac{i}{2 \pi} \int_{\Gamma} R_{z}(f) \mathrm{d} z= & \int_{0}^{\infty} \frac{\phi_{R}(x) \overline{\phi_{R}(y)}}{\left\|\phi_{R}\right\|^{2}} f(y) \mathrm{d} y \\
= & \varphi_{R}(x)\left\langle f, \varphi_{R}\right\rangle . \\
& -717-
\end{aligned}
$$




\section{Philippe Sécher}

Calculons maintenant $\int_{\Pi_{\delta}} R_{z}(f) \mathrm{d} z$ :

$$
\begin{aligned}
\int_{\Pi_{\delta}} R_{z}(f) \mathrm{d} z= & \int_{\infty}^{1} \int_{0}^{\infty} G(x, y ; k+i \gamma) f(y) \mathrm{d} y \mathrm{~d} k+ \\
& +\int_{1}^{\infty} \int_{0}^{\infty} G(x, y ; k-i \gamma) f(y) \mathrm{d} y \mathrm{~d} k \\
= & \int_{1}^{\infty} \int_{0}^{\infty}(G(x, y ; k-i \gamma)-G(x, y ; k+i \gamma)) f(y) \mathrm{d} y \mathrm{~d} k \\
= & \int_{1}^{\infty} \int_{0}^{\infty}(G(x, y ; k-i 0)-G(x, y ; k+i 0)) f(y) \mathrm{d} y \mathrm{~d} k
\end{aligned}
$$

On utilise l'holomorphie de $G$ en dehors de l'axe réel pour faire tendre le contour vers cet axe, ceci correspond à faire tendre $\gamma$ vers zéro, $k+i 0=$ $\lim _{\gamma \rightarrow 0_{+}}(k+i \gamma)$.

Notons pour $k$ tel que $k>1$ :

$$
H(x, y, k)=G(x, y ; k-i 0)-G(x, y ; k+i 0) .
$$

Lemme 3.2. - Pour tout $y$ et $x$ dans $\mathbb{R}^{+}$

$$
H(x, y, k)=\left\{\begin{array}{lc}
-\frac{i}{2 k T(k)} \phi_{1}(x, k) \overline{\phi_{1}(y, k)}-\frac{i}{2 k^{2} L(k)} \phi_{2}(x, k) \overline{\phi_{2}(y, k)} \\
-\frac{i}{2 k T(k)} \phi_{1}(x, k) \overline{\phi_{1}(y, k)} & \text { si } k>c^{2} \\
& \text { si } k \in] 1, c^{2}[.
\end{array}\right.
$$

Démonstration. - $G(x, y ; k-i 0)$ et $G(x, y ; k+i 0)$ s'exprimant à partir des fonctions $T, L, u_{T}^{ \pm}$et $u_{L}^{ \pm}$, pour calculer ces limites on doit distinguer différents domaines pour $k$. Plus particulièrement, on doit situer $k$ par rapport à $c^{2}$, comme on l'a vu à la section 1 où ont été définies les fonctions $L, T, u_{T}^{ \pm}$et $u_{L}^{ \pm}$.

On distingue donc deux domaines pour le calcul de $H$ (définie seulement pour $k>1$ ).

- Premièrement si $k>c^{2}$,

$$
\begin{aligned}
R_{1}(k-i 0) & =R_{1}(k) \\
R_{2}(k-i 0) & =R_{2}(k) \\
\phi_{1}(k-i 0) & =\left(u_{T}^{+}+R_{2} u_{T}^{-}-T R_{1} u_{L}^{-}\right)(k) \\
\phi_{2}(k-i 0)= & \left(u_{L}^{+}+L R_{1} u_{T}^{-}+R_{2} u_{L}^{-}\right)(k) . \\
& \quad-718-
\end{aligned}
$$


Étude spectrale du système différentiel $2 \times 2$ associé à un problème d'élasticité linéaire

Dans ce cas pour $y>x$, on a

$$
G(x, y ; k+i 0)=\frac{i}{2 k^{2} T(k)} \phi_{1}(x, k) \overline{u_{T}^{\bar{T}}(y, k)}+\frac{i}{2 k^{2} L(k)} \phi_{2}(x, k) \overline{u_{L}^{\bar{L}}(y, k)}
$$

et

$$
\begin{aligned}
G(x, y ; k-i 0)= & -\frac{i}{2 k T(k)} \phi_{1}(x, k) \overline{\left(R_{2} u_{T}^{+}+R_{1} T u_{L}^{+}\right)(y, k)}+ \\
& -\frac{i}{2 k L(k)} \phi_{2}(x, k) \overline{\left(-R_{1} L u_{T}^{+}+R_{2}(k) u_{L}^{+}\right)(y, k)} .
\end{aligned}
$$

Alors

$$
H(x, y, k)=\frac{-i}{2 k T(k)} \phi_{1}(x, k) \overline{\phi_{1}(y, k)}-\frac{i}{2 k L(k)} \phi_{2}(x, k) \overline{\phi_{2}(y, k)}
$$

pour $y>x$.

Le problème étant autoadjoint, on a $G(x, y ; z)=\overline{G(y, x ; \bar{z})}$ ce qui entraîne $H(x, y, k)=\overline{H(y, x, k)}$. On a donc

$$
H(x, y, k)=\frac{-i}{2 k T(k)} \phi_{1}(x, k) \overline{\phi_{1}(y, k)}-\frac{i}{2 k L(k)} \phi_{2}(x, k) \overline{\phi_{2}(y, k)}
$$

$\forall x, y \in \mathbb{R}^{+}$pour $k>c^{2}$.

- Deuxièmement si $k \in] 1, c^{2}[$,

$$
\begin{aligned}
& R_{1}(k-i 0)=-\frac{R_{1}(k)}{R_{2}(k)}=\overline{R_{1}(k)} \\
& R_{2}(k-i 0)=\frac{1}{R_{2}(k)}=\overline{R_{2}(k)} \\
& \phi_{1}(k-i 0)=\left(u_{T}^{+}+\bar{R}_{2} u_{T}^{-}-T \bar{R}_{1} u_{L}^{+}\right)(k) \\
& \phi_{2}(k-i 0)=\left(u_{L}^{\bar{L}}+L \bar{R}_{1} u_{T}^{-}+\bar{R}_{2} u_{L}^{+}\right)(k) .
\end{aligned}
$$

Dans ce cas, pour $y>x$, on a

$$
G(x, y ; k+i 0)=\frac{i}{2 k T(k)} \phi_{1}(x, k) \overline{u_{T}^{\bar{T}}(y, k)}+\frac{i}{2 k L(k)} \phi_{2}(x, k) \overline{u_{L}^{+}(y, k)}
$$

et

$$
\begin{aligned}
G(x, y ; k-i 0) & =-\frac{i}{2 k T(k)}\left(\overline{R_{2}} \phi_{1}\right)(x, k) \overline{u_{T}^{+}(y, k)} \\
& +\frac{i}{2 k L(k)}\left(u_{L}^{-}-L \overline{R_{1}} u_{T}^{-}+\overline{R_{2}} u_{L}^{+}(x, k)\right) \overline{u_{L}^{+}(y, k)} .
\end{aligned}
$$




\section{Philippe Sécher}

Alors

$$
H(x, y, k)=-\frac{i}{2 k T(k)} \phi_{1}(x, k) \overline{\phi_{1}(y, k)}
$$

et ceci pour tout $y>x$ d'où, comme dans le premier domaine, en appliquant la formule $H(x, y, k)=\overline{H(y, x, k)}$, on obtient

$$
H(x, y, k)=-\frac{i}{2 k T(k)} \phi_{1}(x, k) \overline{\phi_{1}(y, k)}, \quad \forall x, y \in \mathbb{R}^{+} \text {pour } 1<k<c^{2} .
$$

Le lemme est donc démontré.

Revenons à la démonstration du théorème (3.1). Par ce qui précède, on obtient le résultat suivant :

$$
\begin{aligned}
\frac{i}{2 \pi} \int_{\Pi_{\delta}} R_{z}(f) \mathrm{d} z= & \int_{1}^{\infty} \int_{0}^{\infty} \frac{1}{4 \pi k T(k)} \phi_{1}(x, k) \overline{\phi_{1}(y, k)} f(y) \mathrm{d} y \mathrm{~d} k+ \\
& +\int_{c^{2}}^{\infty} \int_{0}^{\infty} \frac{1}{4 \pi k L(k)} \phi_{2}(x, k) \overline{\phi_{2}(y, k)} f(y) \mathrm{d} y \mathrm{~d} k \\
= & \int_{1}^{\infty} \int_{0}^{\infty} \varphi_{1}(x, k) \overline{\varphi_{1}(y, k)} f(y) \mathrm{d} y \mathrm{~d} k+ \\
& +\int_{c^{2}}^{\infty} \int_{0}^{\infty} \varphi_{2}(x, k) \overline{\varphi_{2}(y, k)} f(y) \mathrm{d} y \mathrm{~d} k
\end{aligned}
$$

On en déduit que pour tout $x$ dans $\mathbb{R}^{+}$et tout $f$ dans $L^{2}\left(\mathbb{R}^{+}\right)$

$$
\begin{aligned}
f(x)= & \varphi_{R}(x) \tilde{f}_{R}+\int_{1}^{\infty} \int_{0}^{\infty} \varphi_{1}(x, k) \overline{\varphi_{1}(y, k)} f(y) \mathrm{d} y \mathrm{~d} k+ \\
& +\int_{c^{2}}^{\infty} \int_{0}^{\infty} \varphi_{2}(x, k) \overline{\varphi_{2}(y, k)} f(y) \mathrm{d} y \mathrm{~d} k .
\end{aligned}
$$

Le théorème est ainsi démontré.

On peut en déduire les corollaires suivants.

Corollaire 3.3.- On a

$$
\Phi^{*} \Phi=\operatorname{Id}_{L^{2}\left(\mathbb{R}^{+}\right)}
$$

Démonstration. - Grâce au théorème 3.1 ; on sait que pour tout $f$ dans $L^{2}\left(\mathbb{R}^{+}\right)$,

$$
\begin{aligned}
f(x)= & \varphi_{R}(x) \tilde{f}_{R}+\int_{1}^{\infty} \int_{0}^{\infty} \varphi_{1}(x, k) \overline{\varphi_{1}(y, k)} f(y) \mathrm{d} y \mathrm{~d} k+ \\
& +\int_{c^{2}}^{\infty} \int_{0}^{\infty} \varphi_{2}(x, k) \overline{\varphi_{2}(y, k)} f(y) \mathrm{d} y \mathrm{~d} k .
\end{aligned}
$$


Étude spectrale du système différentiel $2 \times 2$ associé à un problème d'élasticité linéaire

Or pour $f$ dans $\mathbf{S}(\mathbb{R}) \cap L^{2}\left(\mathbb{R}^{+}\right)$, on a

$$
\begin{aligned}
& \Phi_{1}(f)=\int_{0}^{\infty} \overline{\varphi_{1}(y, k)} f(y) \mathrm{d} y \\
& \Phi_{2}(f)=\int_{0}^{\infty} \overline{\varphi_{2}(y, k)} f(y) \mathrm{d} y
\end{aligned}
$$

et

$$
\Phi_{1}^{\circ}(f)=\int_{0}^{\infty} \overline{\varphi_{1}(y, k)} f(y) \mathrm{d} y .
$$

De plus dans ce cas,

$$
\begin{aligned}
& \Phi_{1}^{*} \Phi_{1}(f)=\int_{c^{2}}^{\infty} \varphi_{1}(x, k) \tilde{f}_{1}(k) \mathrm{d} k \\
& \Phi_{2}^{*} \Phi_{2}(f)=\int_{c^{2}}^{\infty} \varphi_{2}(x, k) \tilde{f}_{2}(k) \mathrm{d} k
\end{aligned}
$$

et

$$
\Phi_{1}^{\circ *} \Phi_{1}^{\circ}(f)=\int_{1}^{c^{2}} \varphi_{1}(y, k) \widetilde{f}_{1}^{\circ}(k) \mathrm{d} k
$$

On a donc, pour tout $f$ dans $\mathbf{S}(\mathbb{R}) \cap L^{2}\left(\mathbb{R}^{+}\right)$,

$$
\begin{aligned}
f & =\Phi_{R}^{*} \Phi_{R}(f)+\Phi_{1}^{\circ *} \Phi_{1}^{\circ}(f)+\Phi_{1}^{*} \Phi_{1}(f)+\Phi_{2}^{*} \Phi_{2}(f) \\
& =\Phi^{*} \Phi(f) .
\end{aligned}
$$

Le corollaire 3.3 est ensuite démontré par densité.

Ce corollaire entraîne l'égalité de Parseval suivante :

$$
\|f\|^{2}=\left|\tilde{f}_{R}\right|^{2}+\left\|\tilde{f}_{1}^{\circ}\right\|_{L^{2}(] 1, c^{2}[)}^{2}+\left\|\tilde{f}_{1}\right\|_{L^{2}(] c^{2}, \infty[)}^{2}+\left\|\tilde{f}_{2}\right\|_{L^{2}(] c^{2}, \infty[)}^{2} .
$$

Énonçons un autre corollaire découlant du théorème 3.1 .

\section{Corollaire 3.4. - L'application}

$$
\Phi: L^{2}\left(\mathbb{R}^{+}\right) \longrightarrow L^{2}(] c^{2}, \infty[) \oplus L^{2}(] c^{2}, \infty[) \oplus L^{2}(] 1, c^{2}[) \oplus \mathbb{C}
$$

est un isomorphisme.

Démonstration. - Avant de commencer la démonstration de ce corollaire rappelons, comme nous l'avons remarqué à la section 2, que le spectre 


\section{Philippe Sécher}

ponctuel de $A, \mathcal{D}$ est réduit à $\left\{\omega_{R}^{2}\right\}$. Aussi si l'on note $\Pi$ la résolution spectrale associée à $A, \mathcal{D}$, on va décomposer $L^{2}\left(\mathbb{R}^{+}\right)$sous la forme

$$
\Pi(]-\infty, \omega_{R}^{2}[) L^{2}\left(\mathbb{R}^{+}\right) \oplus \Pi\left(\left\{\omega_{R}^{2}\right\}\right) L^{2}\left(\mathbb{R}^{+}\right) \oplus \Pi(] \omega_{R}^{2}, \infty[) L^{2}\left(\mathbb{R}^{+}\right) .
$$

La démonstration du théorème est effectuée en trois étapes.

Première étape. - On montre d'abord que l'application

$$
\begin{aligned}
\Pi\left(\left\{\omega_{R}^{2}\right\}\right) L^{2}\left(\mathbb{R}^{+}\right) & \longrightarrow \mathbb{C} \\
f & \longmapsto \widetilde{f_{R}}
\end{aligned}
$$

est un isomorphisme. Ceci est vérifié car $\varphi_{R}$ est la fonction propre associée à la valeur propre $\omega_{R}^{2}$.

Deuxième étape. - On montre ensuite que $\Pi(]-\infty, \omega_{R}^{2}[) L^{2}\left(\mathbb{R}^{+}\right)=$ $\{0\}$ et que l'application

$$
\begin{aligned}
& \Pi(] \omega_{R}^{2}, \infty[) L^{2}\left(\mathbb{R}^{+}\right) \longrightarrow L^{2}\left(\left[1, c^{2}[) \oplus L^{2}\left(\left[c^{2},+\infty[)\right.\right.\right.\right. \\
& \oplus L^{2}\left(\left[c^{2},+\infty[)\right.\right. \\
& f \\
& \longrightarrow \tilde{f}_{1}^{\circ}(k), \tilde{f}_{1}(k), \tilde{f}_{2}(k)
\end{aligned}
$$

est un isomorphisme.

Pour démontrer ce résultat, on va utiliser la théorie de Weyl et Kodaira développée par exemple dans le livre de [DS]. Les résultats énoncés dans ce livre pour des opérateurs scalaires restent vrais pour des opérateurs différentiels matriciels en adaptant les notations (comme l'ont fait [DG]).

Par ] $a, b$ [ désignons soit ] $-\infty, \omega_{R}^{2}$ [, soit ] $\omega_{R}^{2}, \infty[$.

Définissons sur $\mathbb{R}^{+} \times$] $a, b\left[\right.$ la famille $\left(\psi_{i}\right)_{1 \leq i \leq 4}$ de fonctions suivantes :

$$
\begin{aligned}
& \psi_{1}(x, \lambda)=\varphi_{1}(x, \lambda) \\
& \psi_{2}(x, \lambda)=\varphi_{2}(x, \lambda) \\
& \psi_{3}(x, \lambda)=u_{T}^{+}(x, \lambda) \\
& \psi_{4}(x, \lambda)=u_{L}^{+}(x, \lambda) .
\end{aligned}
$$

$\left(\psi_{i}(\cdot, \lambda)\right)_{1 \leq i \leq 4}$ constitue une base de solutions de $(A-\lambda) \psi=0$, continues sur $\left.\mathbb{R}^{+} \times\right] a, b[$. La théorie de Weyl et Kodaira nous permet d'énoncer la proposition suivante. 
Étude spectrale du système différentiel $2 \times 2$ associé à un problème d'élasticité linéaire

Proposition 3.5. - Il existe une unique mesure matricielle positive $\rho=\left(\rho_{i, j}\right)_{i=1, \ldots, 4, j=1, \ldots, 4}$ sur $] a, b[$ telle que

1) pour tout $f \in L^{2}\left(\mathbb{R}^{+}, \mathbb{C}^{2}\right)$, la limite

$$
\begin{aligned}
\widehat{f}(\lambda) & =\lim _{\substack{\varepsilon \rightarrow 0_{+} \\
\delta \rightarrow+\infty}}\left(\int_{\varepsilon}^{\delta} \overline{\psi_{j}(y, \lambda)} f(y) \mathrm{d} y\right)_{j=1,2,3,4} \\
& =\widehat{f}_{1}(\lambda), \widehat{f}_{2}(\lambda), \widehat{f}_{3}(\lambda), \widehat{f}_{4}(\lambda)
\end{aligned}
$$

existe dans l'espace de Hilbert $L^{2}(] a, b[, \rho)$;

2) l'application

$$
\begin{aligned}
T: L^{2}\left(\mathbb{R}^{+}, \mathbb{C}^{2}\right) & \longrightarrow L^{2}(] a, b[, \rho) \\
f & \longmapsto \widehat{f}(\lambda)
\end{aligned}
$$

est partiellement isométrique, surjective de projecteur initial $\Pi(] a, b[)$;

3) l'isomorphisme réciproque est

$$
\begin{aligned}
T^{*}: L^{2}(] a, b[, \rho) & \longrightarrow \Pi(] a, b[) L^{2}\left(\mathbb{R}^{+}, \mathbb{C}^{2}\right) \\
\widehat{f} & \longmapsto \int_{a}^{b} \sum_{i, j=1}^{4} \psi_{i}(x, \lambda) \hat{f}_{j}(\lambda) \rho_{i, j}(\mathrm{~d} \lambda) ;
\end{aligned}
$$

4) pour toute fonction borélienne $\psi(\cdot)$ sur $\mathbb{R}^{+}$dont le support est inclus dans $] a, b[$, on $a$

$$
T \mathcal{D}(\psi(A))=L^{2}(] a, b[, \rho) \cap\left\{\widehat{f}(\cdot) / \psi(\cdot) \widehat{f}(\cdot) \in L^{2}(] a, b[, \rho)\right\}
$$

et

$$
T \psi(A) f=\psi(\lambda) \widehat{f}(\lambda)
$$

Considérons la mesure matricielle $\rho=\left(\rho_{i, j}\right)_{i=1, \ldots, 4, j=1, \ldots, 4}$ sur $] a, b[$ introduite dans la proposition.

$T$ étant alors unitaire, cela nous permet d'obtenir pour tout $f$ de $\Pi(] a, b[) L^{2}\left(\mathbb{R}^{+}\right)$la formule de représentation suivante :

$$
f=\int_{a}^{b} \sum_{i, j=1}^{4} \psi_{i}(x, \lambda) \int_{0}^{\infty} \overline{\psi_{j}(y, \lambda)} f(x) \mathrm{d} x \rho_{i, j}(\mathrm{~d} \lambda) .
$$


Mais grâce au théorème 3.1 , on a, pour tout $f$ dans $\Pi(] a, b[) L^{2}\left(\mathbb{R}^{+}\right)$,

$$
\begin{aligned}
f(x)= & \int_{1}^{\infty} \int_{0}^{\infty} \varphi_{1}(x, k) \overline{\varphi_{1}(y, k)} f(y) \mathrm{d} y \mathrm{~d} k+ \\
& +\int_{c^{2}}^{\infty} \int_{0}^{\infty} \varphi_{2}(x, k) \overline{\varphi_{2}(y, k)} f(y) \mathrm{d} y \mathrm{~d} k
\end{aligned}
$$

$\left(\tilde{f_{R}}\right.$ étant nulle pour $\left.f \in \Pi(] a, b[) L^{2}\left(\mathbb{R}^{+}\right)\right)$. Ce développement nous permet de calculer la mesure matricielle $\rho$,

$$
\begin{aligned}
& \rho_{1,1}(\mathrm{~d} \lambda)=\chi(] 1, \infty[)(\lambda) \mathrm{d} \lambda \\
& \rho_{2,2}(\mathrm{~d} \lambda)=\chi(] c^{2}, \infty[)(\lambda) \mathrm{d} \lambda \\
& \rho_{1,2}(\mathrm{~d} \lambda)=\rho_{2,1}(\mathrm{~d} \lambda)=\rho_{3,3}(\mathrm{~d} \lambda)=\rho_{3,4}(\mathrm{~d} \lambda)=\rho_{4,3}(\mathrm{~d} \lambda)=\rho_{4,4}(\mathrm{~d} \lambda)=0 .
\end{aligned}
$$

Ceci termine la démonstration de ce deuxième point.

Troisième étape. Conclusion. - Les résultats des étapes précédentes nous permettent de conclure que $\Phi$ est une bijection. La démonstration est terminée.

Énonçons maintenant le résultat le plus important de ce paragraphe.

THÉORÈME 3.6. $-\Phi$ est unitaire.

Démonstration. - Grâce au corollaire 3.3 on sait que $\Phi^{*} \Phi=$ Id. Le corollaire 3.4 nous conduit lui à l'existence de $\Phi^{-1}$. De ces deux résultats on en déduit $\Phi^{-1}=\Phi^{*}$, c'est-à-dire que $\Phi$ est unitaire.

On a donc construit un opérateur $\Phi$ unitaire qui vérifie $\Phi A \Phi^{*}=k$, on peut en déduire la résolution spectrale $\Pi$ de $A$ et la nature du spectre.

THÉORÈmE 3.7. $-\forall f, g \in L^{2}\left(\mathbb{R}^{+}, \mathbb{C}^{2}\right)$ et $\forall \alpha \in \mathbb{R}$, on a

$$
\begin{aligned}
& \langle\Pi(]-\infty, \alpha]) f, g\rangle= \\
& =\int_{c^{2}}^{\infty} H(\alpha-k) \tilde{f}_{1}(k) \overline{\tilde{g}_{1}(k)} \mathrm{d} k+\int_{c^{2}}^{\infty} H(\alpha-k) \tilde{f}_{2}(k) \overline{\tilde{g}_{2}(k)} \mathrm{d} k+ \\
& \quad+\int_{1}^{c^{2}} H(\alpha-k) \tilde{f}_{1}^{o}(k) \overline{\tilde{g}_{1}^{\circ}(k)} \mathrm{d} k+H\left(\alpha-\omega_{R}^{2}\right) \tilde{f}_{R} \overline{\tilde{g}_{R}} .
\end{aligned}
$$

Démonstration. - C'est simplement une transformation de la résolution spectrale de l'opérateur de multiplication par $k$ par l'isomorphisme $\Phi$. 
Étude spectrale du système différentiel $2 \times 2$ associé à un problème d'élasticité linéaire

\section{Remarques}

- Le spectre $\sigma_{A}$ de $A$ vaut $\left\{\omega_{R}^{2}\right\} \cup[1, \infty[;$ il est de plus absolument continu sur $\left[1, \infty\right.$ [, de multiplicité 1 sur $\left[1, c^{2}\right]$ et 2 sur $] c^{2}, \infty[$.

- Ce spectre est de même nature que celui de l'opérateur de Laplace dans un milieu stratifié. En effet, si on regarde les résultats énoncés dans ce cas par Wilcox [W], on retrouve bien une partie ponctuelle et une partie absolument continue de multiplicité 1 entre les deux vitesses et 2 pour la partie supérieure à la plus grande.

- Les deux problèmes sont pourtant totalement différents. Dans un cas, les deux vitesses et la présence de valeurs propres isolées proviennent de la stratification. Dans l'autre, la présence des deux vitesses est due à l'opérateur d'élasticité et la présence du spectre purement ponctuel provient du domaine considéré, en particulier de la présence du bord libre.

- L'étude de $\Delta_{e}$ sur $\mathbb{R}_{+}^{n}$ avec condition de Neumann sur le bord se déduit de ces résultats à l'aide des transformations inverses de celles effectuées pour découpler le problème. Les techniques sont les même que celles employées par Wilcox [W].

\section{Références}

[A] Achenbach (J. D.) .- Wave propagation in elastic solids, North Holland Amsterdam, Applied Mathematics and Mechanics 16 (1973).

[B] BREKHOVSKIKH (L. M.) .- Waves in layered media (second edition), Academic Press (1980).

[BS] Berezin (F. A.) et Shubin (M. A.) .- The Schrödinger equation, Kluwer. Acad. Pub. (1991).

[CP] Chazarain (J.) et Piriou (A.) .- Introduction à la théorie des équations aux dérivées partielles linéaires, Gauthier-Villars (1981).

[DG] Dermenjian (Y.) et Guillot (J.-C.) .- Scattering of elastic waves in a perturbed isotropic half space with a free boundary. The limiting absorption principle, Mathematical Methods in the Applied Sciences 10 (1988), pp. 87 124.

[DS] DUNFORD (N.) et SChWARTZ (J. T.) .- Linear operators, Part II: spectral theory, Intersience (1963).

[EJP] EwING (M.), JARDESKY (W.) et Press (F.) .- Elastic waves in layered media, Mac Graw-Hill (1957).

[LP] Leray (J.) et Pecker (A.) .- Calcul explicite du déplacement ou de la tension du demi-plan élastique, isotrope et homogène soumis à un choc en son bord, J. Math Pures. et Appl. 70 (1991), pp. 489-511. 
[R] RAYLEIGH (J. W. STRUTT, LORD) .- On waves propagated along the plane surface of an elastic solid, Sci. Pa. II 441 (1886).

[Se] SÉCHER (P.) . - Étude théorique du comportement dynamique d'un corps de chaussées, Thèse de doctorat. Université de Nantes (1995).

[Sh] SHUBIN (M.) .- Spectral theory of elliptic operators on non compact manifolds, Méthodes semi-classiques 1, École d'été (Nantes, juin 1991); Astérisque 207 (1992), pp. 35-108.

[So] SogA (H.) .- Asymptotic solutions of the elastic wave equation and their applications, Bull. Fac. Educ., Ibaraki Univ. (Nat. Sci.) 38 (1989), pp. 9-16.

[SSo] Shibata (Y.) et Soga (H.) .- Scattering theory for the elastic wave equation, Publ. RIMS Kyoto Univ. 25 (1989), pp. 861-887.

[W] WILCOX (C. H.) .- Sound propagation in stratified fluids, Springer Verlag New-York, Applied Mathematical Sciences 50 (1984). 